\title{
Application Analysis of
}

\section{The Data Visualization Technology in Sports Equipment}

\author{
Liu Shuyi, Liu Yimengqi* \\ Sports Engineering Key Laboratory of State Sport General Administration,
} Hubei Collaborative Innovation Center of Athletic Interference and Health Promotion,

\author{
School of Sports Engineering and Information Technology, \\ Wuhan Physical Education University, \\ Wuhan, Hubei, 430079 \\ *email 369202312@qq.com,*463672297@qq.com
}

\begin{abstract}
With the rapid development and wide spread of sports fitness equipment, the individual sports data, such as the sports time, heart rate, blood pressure, mileage calculation, consumption quantity of heat etc., which is recorded by the sports equipment, is becoming more and more enormous. At the same time, data visualization technology, born in the era of big data, provides a good foundation for the further development of fitness equipment. Through the introduction of data visualization technology, this article probes into the application and future direction of the data visualization technology. With the combination of the data visualization technology and the sports equipment, this article also provides suggestions for the scientific fitness guidance and the development of sports industry.
\end{abstract}

Keywords: Data visualization technology; application analysis; sports equipment.

\section{Introduction}

In 2014, the State Council allotted "Opinions on Accelerating the Development of Sports Industry and Promoting Sports Consumption", proposing that the overall scale of China's sports industry will exceed 5 trillion yuan in 2025. At present, in the case that the sports industry is only around 500 billion yuan, one key to achieve this goal is that sports goods manufacturing enterprises continue to adopt new technologies, develop new products and attract new consumers. The future growth point of the domestic sports equipment manufacturing is the development of intelligent fitness equipment, which is also the major concept and strategic direction on product innovation and market development for the sports equipment industry.

Based on the data visualization technology, we can make these data more intuitive in the form of two-dimensional or three-dimensional, so that the data is more likely to be explained. At the same time, with the aid of data analysis technology, we can dig out the real valuable information from these data and provide the information to the relevant decision makers, which can make the data fully utilized.

\section{Application Industry of Data Visualization Technology}

\subsection{Government--To realize the scientific decision-making and effective governance}

The data visualization application enables the government to make timely, efficient and accurate management means, management decision-making, multifaceted layout and regulation in a short period of time, relying on the data. Moreover, the data visualization can help the government to predict the social problems, dig out potential demands, make scientific forecast, formulate relevant emergency warning plan and plan developing policies etc., so as to improve the government's management ability, service efficiency and build a new type of service government. 


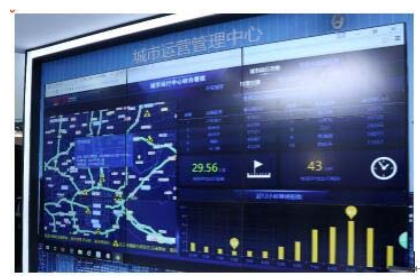

Chart 1 Achieving the scientific decision-making and effective governance through the data visualization technology

\subsection{Finance--To strengthen the risk control and real-time supervision}

Under the fierce competition of today's Internet finance, market situation is changing and the financial industry faces many challenges. To calmly face the market competitive challenges and improve enterprise's competitiveness requires managers to make precise and scientific management based on the big data.

Data visualization can do dynamic real-time control around the daily business, effective regulation about the customer number and the amount of borrowing data, help enterprises to realize the data real-time monitoring, and thus strengthen the supervision and management of the market. Based on the core data of multidimensional analysis and contrast, it guides the company to scientifically adjust the operating strategy, make development direction and constantly improve the company's ability and competitiveness of the risk control management.

\subsection{APP--To do the application analysis and operation}

For a certain APP, the data display and analysis is a necessary content. Clearly showing the analysis data can let users understand their current task quickly, make the APP more intuitive and efficient, and even have a friendly interaction between users and the data. The striking pie chart, bar chart and line chart can be used in all kinds of APP mobile applications, and display the data in a convenience and non-disturbance mode. The beautiful data gram can effectively and succinctly provide users with the necessary information.

\section{Application Prospect for the Data Visualization in Sports Equipment}

\subsection{Application of the image technology used in sports equipment}

Spinning, with integrating intelligent, entertainment and technology, is one of the apparatus with large energy consuming in the gym. It not only steps down the mileage, calories and other functions, but also can real-time monitor the heart rate changes. The data visualization technology can measure the data shorter and accurate. Cooperating with APP, the data visualization technology can make you know your heart rate changes and the exercise intensity represented by your current heart rate.

Rowing apparatus, also named the rowing machine, is a kind of equipment simulating the rowing movement. It is designed to exercise part of the body's muscle, especially the muscles on the arm, back and abdomen, which can effectively reduce the excess fat and achieve the goal of losing weight and bodybuilding. Rowing machine monitor can provide the training distance, speed, pace, power and the number of calories for each time, and also provide five data display options through the data visualization technology: "the total data", "the strength curve", "the bar diagram", "the big fonts" and "the speed boat/the speedometer".
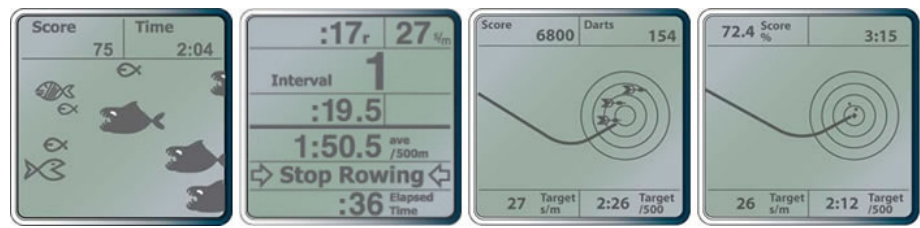

Chart 2 Onceopt2 PM5 Four graphical games provided by the ability monitor

At present, the data can be showed by the technology of image, using image, curve, two-dimensional graphics, solid figure and animation, and it can be analyzed with the visualization technology through its patterns and mutual relationships. It greatly speeds up the process of data analysis and uses the large data produced by the sports equipment more efficiently. It realizes the image communication between human beings and data so that people can observe the phenomenon 
implied in data, thus providing a powerful tool to discover and understand the scientific rules. It also can guide and control the process of calculation and programming, and observe the effects by interactive means based on its changing process.

\subsection{Impact of the data visualization technology on outdoor sports equipment industry}

Outdoor sports equipment refers to a series of sports equipment which is set on the community, parks and other public places for the public fitness and entertainment. As the most important means for the national fitness project, the outdoor fitness route engineering has brought great vitality to the development of mass sports in China. The concept of sports fitness has ever deepened into the Chinese mind, and the healthy and scientific life style is becoming more and more important in the daily life of Chinese people. However, after the investigation and study, the domestic outdoor sports equipment at present still has certain problems such as the singleness of function, low degree of application in the Internet of things, and non-available scientific fitness guidance.

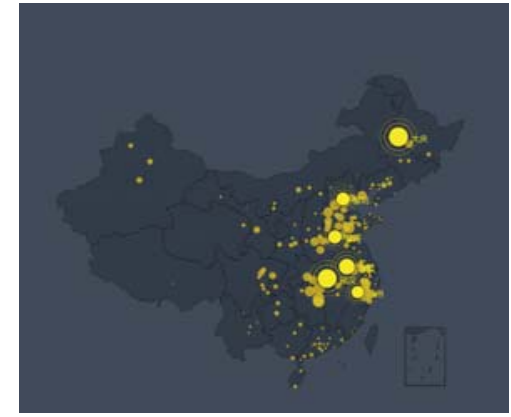

Chart 3 Distribution of the national usage of the outdoor sports equipment(the analog data)

To combine the outdoor sports equipment with the Internet of things and mobile Internet integration, realize the national data synchronization, use the data visualization technology can improve the level of automation, and intelligent health information services. The data visualization technology transforms massive data into statics or dynamic images or graphic presentations in front of people, relying on the enormous processing ability of computer, the basic algorithms of computer graphics and the visualization algorithm. And it controls the data extraction and image display through interactive methods, in order to make the invisible phenomenon implied in data become visible, and provide the scientific basis for the government to make policies relevant to the sports industry.

\subsection{Application of the virtual reality technology in sports equipment}

The development of the data visualization technology has greatly promoted the development of the virtual reality technology. The combination of the two, can greatly enhance the user's "immersion" and "presence". Such kind of immersion and presence would create a feeling of being personally on the scene, which inevitably deepen the acquaintance of information and thus strengthen the memory. Therefore, the data visualization technology has a good application prospect in the virtual reality field.

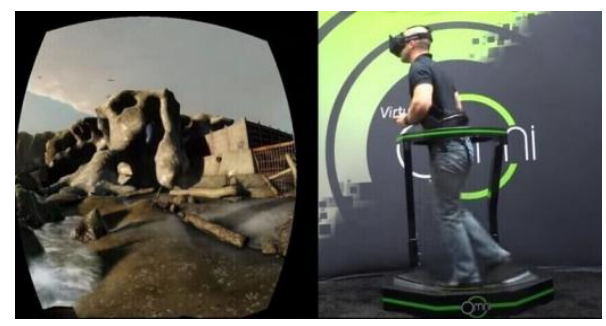

Chart 4 Virtual Omni Virtual reality treadmills

Because they can't persist in to taking exercise, most people lose heart in the hard exercise. If the virtual reality technology can be brought into the sports equipment, it will analyze the sports talent's psychological change and the movement posture change at any time, based on the Internet of things and technology of the big data cloud platform. For many years, sports equipment companies are trying to improve traditional sports equipment such as treadmills which bring about the boring feeling. With the advent of the virtual reality technology, this problem will finally be solved. We can believe 
that a good technology will continually progress, update and become mature, and it can improve more applications and the market value.

\section{Conclusions}

This article narrates the data visualization technology, aimed at analyzing the application of this technology in sports industries. It takes advantage of the sports equipment data, so as to better serve the users, and provide valuable information. At present, science and technology promote the development of the fitness equipment industry, showing a very broad market prospect, and thus a large amount of data will come into being. How to effectively process and interpret the large amounts of data which contain different information will be the huge challenge to human beings. At the same time, it is also the attractive application prospect provided by the data visualization technology.

\section{References}

[1] Lei hui, Lao Tianyi, Liu zhen, et. Physical Data Visualization Review [J]. Journal of Computer-aided Design and Computer Graphics, 2015, 27(9):1605-1616

[2] Ren Yonggong, Yu Ge. Research and Progress of the Data Visualization Technology [J]. Computer Science, 2004, 31(12):92-96

[3] Ren Yonggong. Research on the Data Visualization Method and Related Technology Facing the Clustering Data [D]. Northeastern University, 2006

[4] Shi Haosu, Han Lina. Data Visualization Technology and Its Application Prospect[C]// National Academic Exchange Conference on Automation Technology (I), 2005

[5] Thorvaldsdóttir H, Robinson J T, Mesirov J P. Integrative Genomics Viewer (IGV): high-performance genomics data visualization and exploration[J]. Briefings in Bioinformatics, 2013, 14(2):178-192

[6] Venna J, Peltonen J, Nybo K, et al. Information Retrieval Perspective to Nonlinear Dimensionality Reduction for Data Visualization.[J]. Journal of Machine Learning Research, 2010, 11(1):451-490

[7] Zhang Hao, Guo Can. Research on the Data Visualization Technology Trend and Classification [J]. Software Guide, 2012, 11(5):169-172

[8] Zeng You. The Concept Research of Data Visualization on the Big Data Era Background [D]. Zhejiang University, 2014 